\title{
Urine Osmolality and Renal Outcome in Patients with Chronic Kidney Disease: Results from the KNOW-CKD
}

\author{
Mi Jung Lee ${ }^{a}$ Tae Ik Chang ${ }^{b}$ Joongyub Lee ${ }^{c}$ Yeong Hoon Kim ${ }^{d}$ \\ Kook-Hwan Ohe Sung Woo Lee ${ }^{\mathrm{f}}$ Soo Wan Kim ${ }^{\mathrm{g}}$ Jung Tak Park ${ }^{\mathrm{h}}$ \\ Tae-Hyun Yoo ${ }^{\text {h }}$ Shin-Wook Kang ${ }^{\text {h }}$ Kyu Hun Choi ${ }^{\text {h }}$ Curie Ahn ${ }^{\mathrm{e}}$ \\ Seung Hyeok Han ${ }^{\text {h }}$

\begin{abstract}
aDepartment of Internal Medicine, CHA Bundang Medical Center, CHA University, College of Medicine, Seongnam, Republic of Korea; ${ }^{b}$ Department of Internal Medicine, National Health Insurance Service Medical Center, Ilsan Hospital, Goyangshi, Republic of Korea; cDepartment of Prevention and Management, Inha University Hospital, Inha University School of Medicine, Incheon, Republic of Korea; ${ }^{d}$ Department of Internal Medicine, Inje University, Pusan Paik Hospital, Busan, Republic of Korea; ${ }^{e}$ Department of Internal Medicine, Seoul National University, Seoul, Republic of Korea; ${ }^{f}$ Department of Internal Medicine, Nowon Eulji Medical Center, Eulji University, Seoul, Republic of Korea; ${ }^{9}$ Department of Internal Medicine, Chonnam National University Medical School, Gwangju, Republic of Korea; h Department of Internal Medicine, College of Medicine, Institute of Kidney Disease Research, Yonsei University, Seoul, Republic of Korea
\end{abstract}

\section{Keywords}

Chronic kidney disease · Estimated glomerular filtration rate $\cdot$ Outcome $\cdot$ Urine osmolality

\begin{abstract}
Background: Urine osmolality indicates the ability of the kidney to concentrate the urine and reflects the antidiuretic action of vasopressin. However, results about the association between urine osmolality and adverse renal outcomes in chronic kidney disease (CKD) are conflicting. We investigated the association between urine osmolality and adverse renal outcomes in a nationwide prospective CKD cohort. Methods: A total of 1,999 CKD patients were categorized into 3 groups according to their urine osmolality tertiles. Primary outcome was a composite of $50 \%$ decline in the estimated glomerular filtration rate (eGFR), initiation of dialysis, or kidney transplantation. Results: During a mean follow-up of $35.2 \pm 19.0$ months, primary outcome occurred in 432 (21.6\%) patients; 240 (36.4\%), 162 (24.3\%), and 30 (4.5\%) in the lowest, middle, and highest tertiles, respectively. Low urine osmolality was independently associated with a greater risk of CKD progression (hazard ratio $[\mathrm{HR}], 1.71$; $95 \%$ confidence interval $[\mathrm{Cl}]$, 1.12-2.59). This association was particularly evident in patients with CKD stages 3-4 (per 10 mosm $/ \mathrm{kg}$ decrease; $\mathrm{HR}, 1.02 ; 95 \% \mathrm{Cl}, 1.00-1.03)$. Adding urine osmolality to a base model with
\end{abstract}




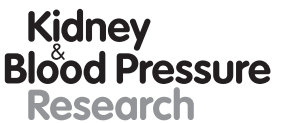

Kidney
Blood Pressure
Research

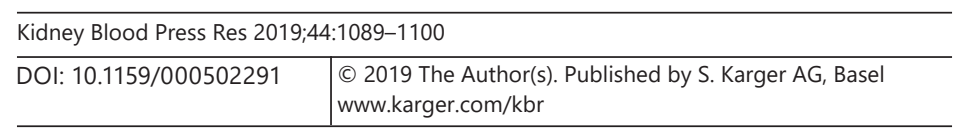

Lee et al.: Urine Osmolality and Renal Outcome in CKD

conventional factors significantly increased the ability to predict CKD progression (C-statistics, 0.86; integrated discrimination improvement [IDI], 0.021; both $p<0.001)$. However, adding both urine osmolality and eGFR did not further improve the predictive ability compared with the addition of eGFR only (C-statistics, $p=0.29$; IDI, $p=0.09$ ). Conclusions: Low urine osmolality was an independent risk factor for adverse renal outcomes in CKD patients, but its predictive ability did not surpass eGFR. Thus, kidney function should be considered while interpreting the clinical significance of urine osmolality.

(C) 2019 The Author(s)

Published by S. Karger AG, Basel

\section{Introduction}

The medical, social, and economic burden from chronic kidney disease (CKD) has been increasing continuously, which poses a worldwide challenge to public health [1-3]. A higher risk of cardiovascular events and all-cause mortality are significantly associated with CKD [4]. Hence, it is important to identify patients who are at high risk for CKD and its progression.

Although urine concentration is the main function of the kidney tubules, the prognostic impact of urine osmolality on adverse renal outcomes has not been fully explored [5-8]. Hebert et al. [5] studied whether high fluid intake (evaluated by urine osmolality and urine volume) was beneficial in delaying kidney disease progression in the post hoc analysis of the Modification of Diet in Renal Disease (MDRD) study [5]. In their study, low urine osmolality was significantly associated with a greater decline in glomerular filtration rate (GFR), regardless of polycystic kidney disease (PKD). They suggested 2 possible explanations for this finding: (1) low urine osmolality itself might cause nephron damage, leading to a decline in kidney function, or (2) low urine osmolality might be an indicator of a urine-concentrating defect due to fast deterioration of the kidney function. On the contrary, a cohort study from the Consortium for Radiologic Imaging Studies of Polycystic Kidney Disease (CRISP) revealed that high urine osmolality was associated with a greater baseline GFR but a steeper decline in GFR in PKD patients [6]. They presumed that high urine osmolality could reflect a high vasopressin effect, which is deleterious to the kidney [6]. Another cohort study of CKD patients also indicated that higher urine osmolality was associated with a higher risk of dialysis [7]. These conflicting results from previous studies led us to investigate the association between urine osmolality and adverse kidney outcomes in CKD patients. Therefore, we examined the prognostic value of urine osmolality on CKD progression in a nationwide prospective cohort of CKD patients in Korea.

\section{Materials and Methods}

\section{Study Design and Participants}

The Korean Cohort Study on the Outcome of Chronic Kidney Disease Patients (KNOWCKD) is an ongoing, nationwide, prospective cohort study including nondialysis patients with CKD stages 1-5 in Korea (NCT01630486 at http://www.clinicaltrials.gov). The detailed design and methods have been described previously [9]. Of the 2,238 participants initially recruited, we excluded 239 participants. Finally, 1,999 patients were enrolled in this study (online suppl. Fig. 1; for all online suppl. material, see www.karger.com/doi/10.1159/000502291).

\section{Data Collection}

Demographics and laboratory data were retrieved from the electronic data management system (PhactaX, Seoul, Korea) with assistance from the Division of Data Management at the 


\section{Kidney \\ Blood Pressure \\ Research}

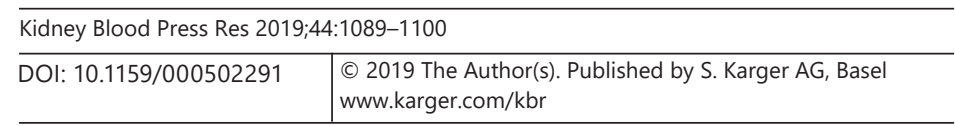

Lee et al.: Urine Osmolality and Renal Outcome in CKD

Seoul National University Medical Research Collaborating Center. Demographics, including the cause of CKD, comorbidities, and medication history, were collected during study enrollment. The age-adjusted Charlson comorbidity index was calculated [10]. Laboratory variables were measured using an 8-h fasting blood sample at the hospital laboratory of each participating center. Serum creatinine was measured by an isotope dilution mass spectrometry-traceable method at the central laboratory (Lab Genomics, Seoul, Korea). The CKD Epidemiology Collaboration equation was used to determine the estimated GFR (eGFR) [11]. CKD stages 1-5 were categorized based on the Kidney Disease Improving Global Outcome guidelines [12].

\section{Urinalysis}

In the fasting status, the first voided urine was collected, and samples were immediately sent to the central lab for measurement of urine osmolality and proteinuria. Urine osmolality (expressed as mosm/kg) was measured using a freezing point osmometer (Micro-Osmette $5004^{\circledR}$; Precision Systems Inc., Natick, MA, US). Urinary protein excretion was determined as urinary protein-to-creatinine ratio (UPCr, g/g). If the participants agreed to 24-h urine collection, they were instructed by research coordinators to collect all voided urine samples at home. In 1,850 participants, the 24-h urine volume was measured and expressed as urine volume per day (mL/day).

\section{Follow-Up and Outcome Assessment}

Participants were followed-up until December 31, 2016. The primary outcome was a composite of $50 \%$ decline in eGFR and the necessity for initiation of renal replacement therapy, including dialysis or renal transplantation.

\section{Statistical Analysis}

Statistical analyses were performed using R (R Foundation for Statistical Computing, Vienna, Austria; www.r-project.org). Continuous variables were expressed as the mean \pm standard deviation or median (interquartile range). Categorical variables were expressed as numbers (\%). Patients were divided into 3 groups according to the urine osmolality tertiles. To compare the baseline characteristics according to urine osmolality tertiles, ANOVA with Bonferroni or Kruskal-Wallis test was used for continuous variables, and a $\chi^{2}$ test was used for categorical variables. The association between urine osmolality and baseline characteristics was evaluated by uni- and multivariable linear regression analyses. Gray's test was employed to estimate the cumulative renal survival rates. Survival time was defined as the time interval between baseline and the first onset of a renal endpoint. To test the independent prognostic value of urine osmolality on the primary outcome, multivariable cause-specific hazard models were constructed including the significant variables $(p<0.05)$ in univariate analysis, and incremental adjustment was performed. Patients who were lost to follow-up were censored at the date of the last examination. Twenty-two deaths that occurred before reaching the primary outcome were treated as a competing risk and as censoring in causespecific hazards models. The prognostic value of urine osmolality was further ascertained using the calculated cutoff value $(474 \mathrm{mosm} / \mathrm{kg}$ ), which was determined by maximally selected rank statistics. Restricted cubic splines were used to reveal the association between urine osmolality as a continuous variable and the hazard ratio (HR) for a renal event. Subgroup analyses were performed according to age, gender, CKD stages (stages 1-2 or 3-4), 24-h urine volume $(<1,500$ or $\geq 1,500 \mathrm{~mL} /$ day $)$, the cause of CKD, and use of diuretics. The rate of renal function decline per year was determined by the slope of eGFR obtained from a generalized linear mixed model. Finally, we compared the predictive ability of urinary osmolality with other prognostic parameters. To this end, we created 4 models and calculated C-statistics, the area under the receiver-operating characteristics curves (AUC), categorical net reclassifi- 


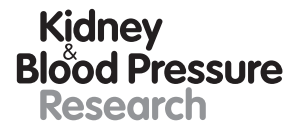

Kidney
Blood Pressure
Research

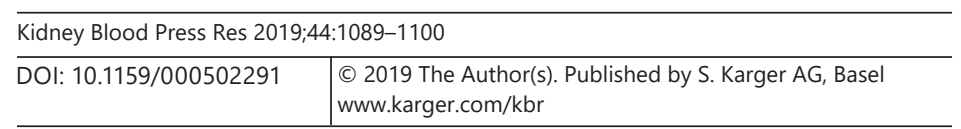

Lee et al.: Urine Osmolality and Renal Outcome in CKD

cation improvement (cNRI), and integrated discrimination improvement (IDI) for each model. Model 1 included conventional factors. Models 2 and 3 were constructed by adding urine osmolality and eGFR to model 1, respectively. Finally, model 4 included both urine osmolality and eGFR in addition to model 1. Finally, model 4 included both urine osmolality and eGFR in addition to model 1. $p<0.05$ was considered statistically significant.

\section{Results}

\section{Baseline Characteristics}

The baseline characteristics according to urine osmolality tertiles are presented in Table 1. The mean age of participants was $53.8 \pm 12.1$ years, and 1,224 (61.2\%) patients were men. The mean baseline eGFR was $50.3 \pm 30.0 \mathrm{~mL} / \mathrm{min} / 1.73 \mathrm{~m}^{2}$, and $1,223(61.2 \%)$ patients had proteinuria $>0.3 \mathrm{~g} / \mathrm{g}$. The mean urine osmolality was $486.7 \pm 160.1 \mathrm{mosm} / \mathrm{kg}$. The mean urine osmolality in the low, middle, and high tertiles was 324.2, 466.3, and 666.8 mosm/kg, respectively. Age, age-adjusted Charlson comorbidity index, systolic blood pressure, serum phosphorous, parathyroid hormone, UPCr, and urine volume significantly decreased as the urine osmolality increased. In contrast, body mass index, serum albumin, calcium, low-density lipoprotein cholesterol, and eGFR significantly increased as the urine osmolality increased. Patients with higher urine osmolality were less likely to be women, diabetics, and on diuretics.

\section{Association between Urine Osmolality and Clinical Characteristics}

Factors that significantly correlated with urine osmolality are presented in supplemental Table 1. In multivariable linear regression analyses, urinary osmolality had an independent positive association with age, body mass index, and baseline eGFR, but an inverse association with parathyroid hormone levels, UPCr, and urine volume (online suppl. Table 1 and suppl. Fig. 2). In particular, the correlation coefficient was highest between urine osmolality and eGFR.

\section{Independent Prognostic Value of Urine Osmolality for Adverse Renal Outcomes}

During a mean follow-up of $35.2 \pm 19.0$ months, primary outcome occurred in 432 (21.6\%) patients. The incidence of primary outcome for the tertiles was $36.4 \%$ ( 240 patients), $24.3 \%$ (162 patients), 4.5\% (30 patients) in the lowest, middle, and highest tertiles, respectively. The crude HRs were 10.87 (95\% confidence interval [CI], 7.47-15.82) and 5.95 (95\% CI, 4.04-8.76) in the lowest and middle tertiles of urine osmolality compared with the highest tertile (Table 2). The results were identical in a multivariable cause-specific hazard model adjusted for demographics and laboratory variables (model 1). This association was much attenuated after further adjustment of eGFR (model 2), but a significantly increased risk of CKD progression in the lowest tertile remained (HR, 1.71; 95\% CI, 1.12-2.59). When urine volume was added to the model (model 3), the lowest tertile was significantly associated with a 1.66-fold increased risk of CKD progression compared to the highest tertile (HR, 1.66; $95 \% \mathrm{CI}, 1.08-2.54)$. Using the calculated cutoff value of urine osmolality, higher risk of low urine osmolality for CKD progression remained significant ( $<474 \mathrm{mosm} / \mathrm{kg}$; HR, 1.62; 95\% CI, $1.25-2.10 ; p<0.001 ; \geq 474 \mathrm{mosm} / \mathrm{kg}$ as reference). Furthermore, the results were consistent when urine osmolality was treated as a continuous variable (per $10 \mathrm{mosm} / \mathrm{kg}$ decrease, HR, 1.02; 95\% CI, 1.01-1.03; $p=0.002$ ). The adjusted cumulative adverse renal events were also significantly higher in the lowest tertile than in the highest tertile $(p<$ 0.001; Fig. 1). Spline regression analyses showed that HRs for CKD progression increased as urine osmolality decreased (Fig. 2), but the magnitude of HRs was also attenuated in the fully adjusted model. 
Kidney
Blood Pressure

Research
Kidney Blood Press Res 2019;44:1089-1100

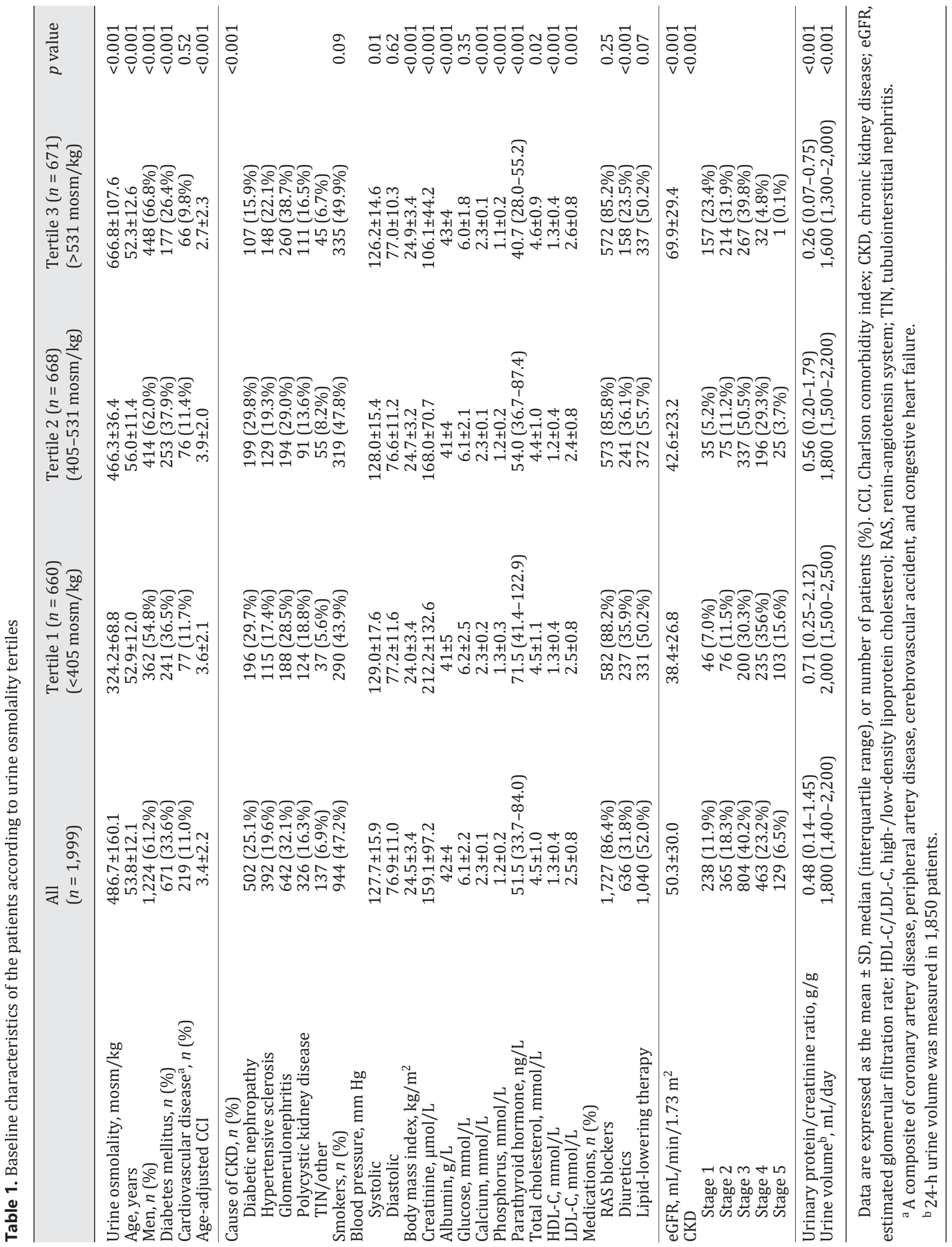


Fig. 1. Adjusted cumulative adverse renal events according to the tertiles of urine osmolality. The adjusted cumulative adverse renal events were significantly higher in the lowest than the highest tertile $(p<0.001)$.

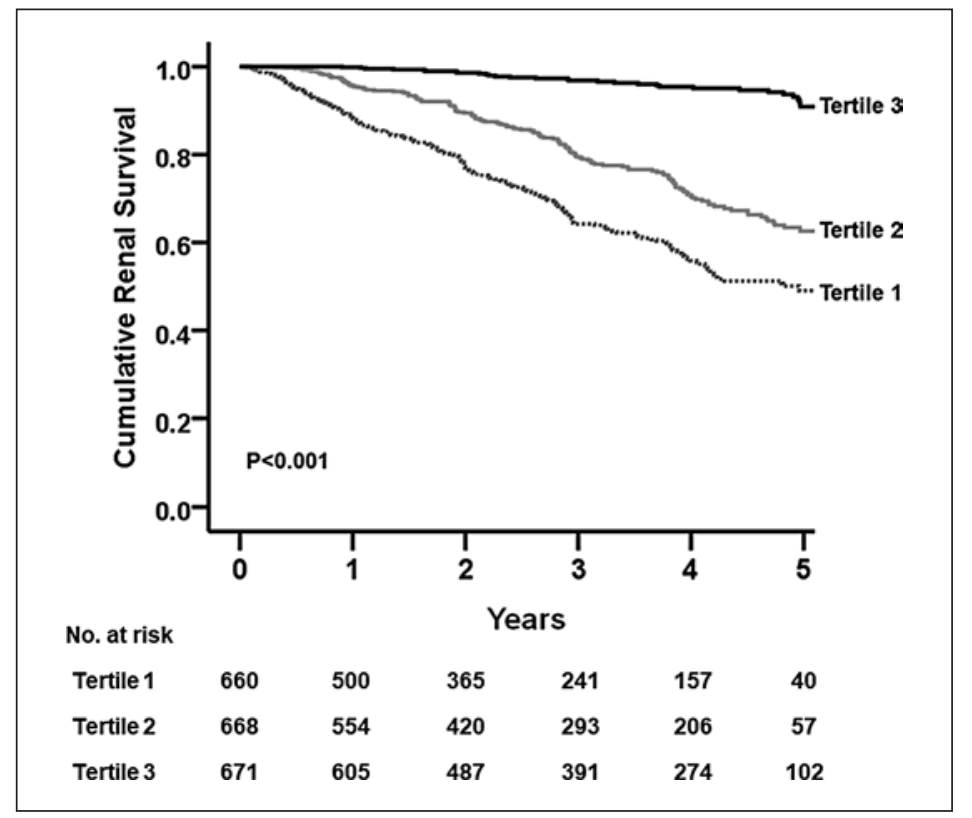

(A)

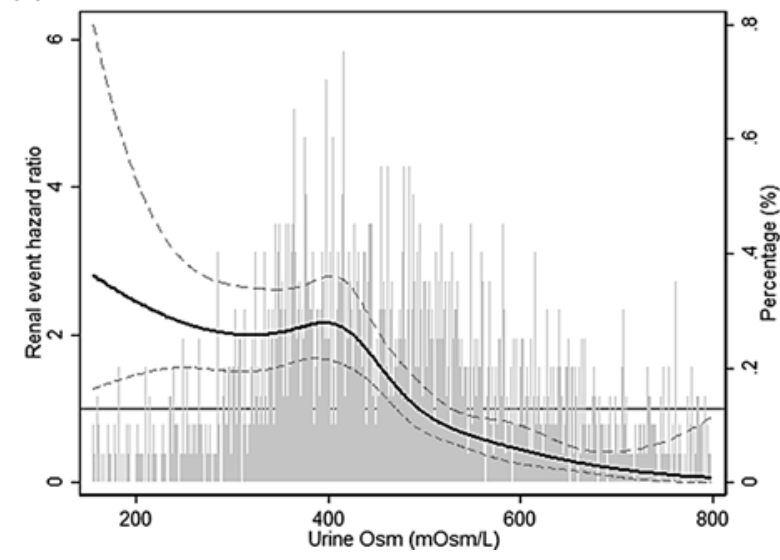

(B)

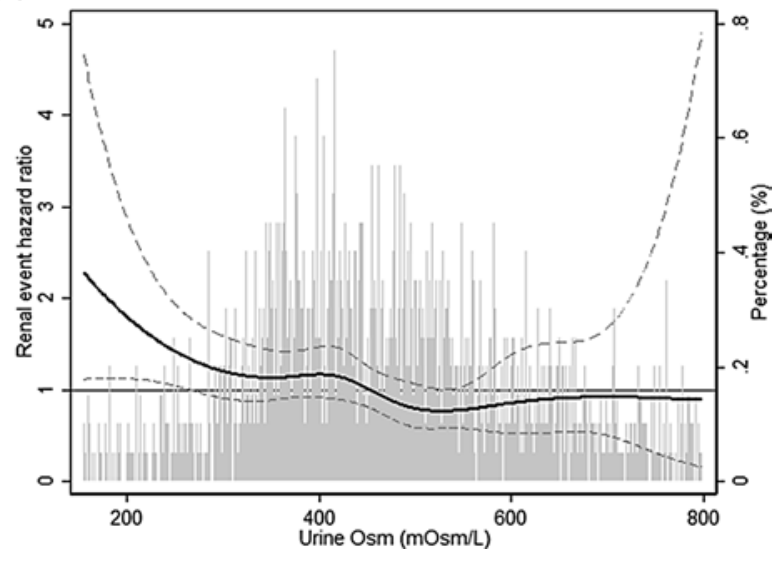

Fig. 2. Cubic spline analyses of the association between urine osmolality and renal event. The solid line represents the crude (a) and fully adjusted (b) hazard ratio, and the dashed lines represent the 95\% confidence intervals. The fully adjusted model included sex, age, diabetes mellitus, cardiovascular disease, smoking history, systolic blood pressure, body mass index, serum albumin, parathyroid hormone, low-density lipoprotein cholesterol, renin-angiotensin system blocker use, diuretic use, urinary protein/creatinine ratio, estimated glomerular filtration rate, and 24-h urine volume.

\section{Subgroup Analyses}

There were no significant interactions in the subgroups stratified by age, gender, CKD causes, diuretic use, and urine volume (Fig. 3). In contrast, urine osmolality had a significant interaction with kidney function. The prognostic value of low urinary osmolality was valid only in patients with advanced CKD (per 10 mosm/kg decrease; CKD stages 3-4, HR, 1.02; 95\% CI, 1.00-1.03; $p=0.01$ ). 
Fig. 3. Adjusted hazard ratio (HR) of urine osmolality for primary outcome according to subgroups including age, sex, cause of chronic kidney disease (CKD), diuretic use, CKD stages, and 24-h urine volume (UV). Adjusted HR was calculated per 10 mosm $/ \mathrm{kg}$ urine osmolality decrease. Adjusted model included sex, age-adjusted Charlson comorbidity index, smoking history, systolic blood pressure, body mass index, serum albumin, parathyroid hormone, low-density lipoprotein cholesterol, renin-angiotensin system blocker and diuretic use, urinary protein/creatinine ratio, and estimated glomerular filtration rate. Subgroup analyses according to CKD stages and 24-h UV were performed in 1,870 and 1,850 patients, respectively. $\mathrm{CI}$, confidence interval; DN, diabetic nephropathy; GN, glomerulonephritis; PKD, polycystic kidney disease.

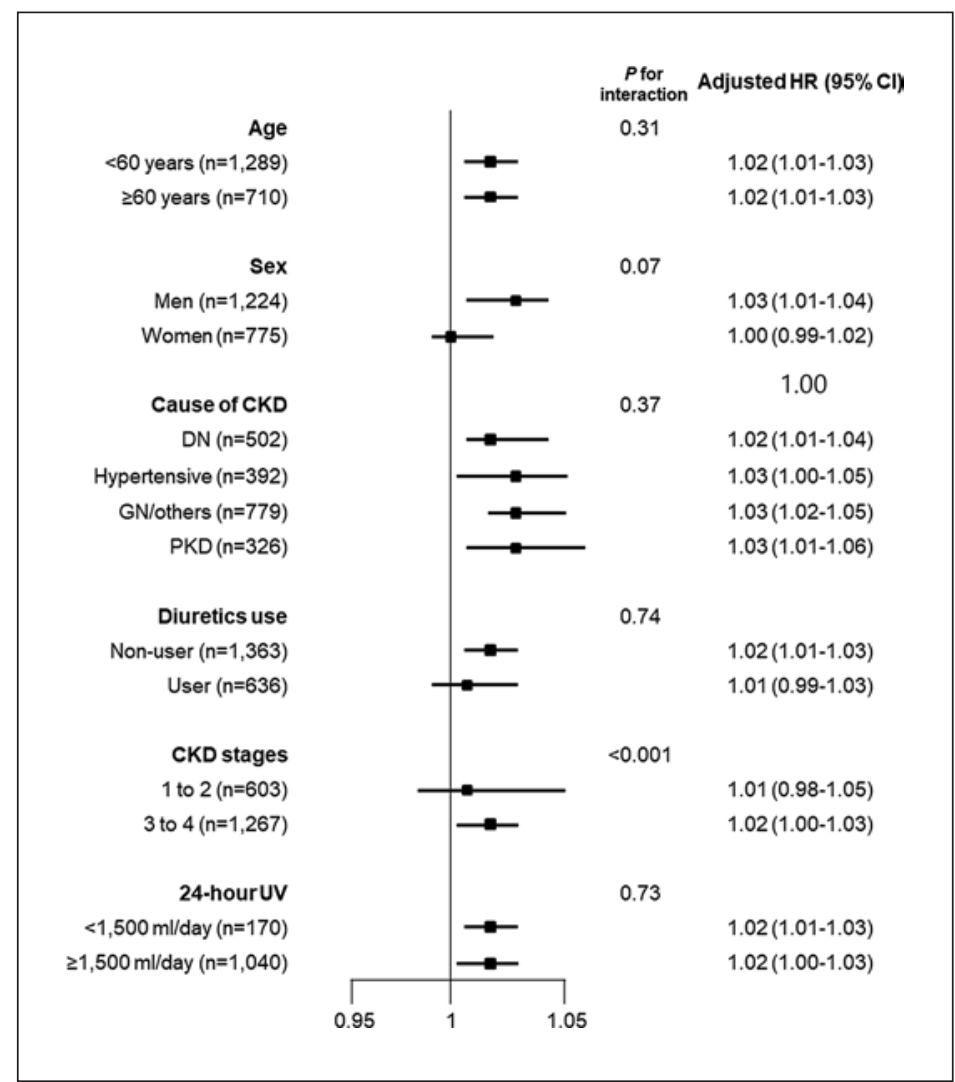

eGFR Decline Rate according to Urine Osmolality

To substantiate our findings further, we compared the slopes of eGFR decline according to the tertiles of urine osmolality (online suppl. Table 2). In line with the results of competing risk models, kidney function declined faster in the lowest tertile of urine osmolality than in the highest tertile.

Predictive Ability of Urine Osmolality

The predictive ability of urine osmolality for CKD progression was tested using C-statistics, AUC, cNRI, and IDI (Table 3). Adding urine osmolality or eGFR to model 1 significantly increased these indices compared to model 1 with conventional factors only (C-statistics, both $p<0.001$; AUC, $p=0.01$ in model 2 and $p<0.001$ in model 3). However, the prediction indices of the urine osmolality-added model were significantly lower than those of the eGFRadded model. In addition, inclusion of both urine osmolality and eGFR (model 4) did not further improve these values compared with eGFR alone (model 3). Only cNRI was improved when both urine osmolality and eGFR were added, but the magnitude of the increase in cNRI was small.

\section{Discussion}

In this study, we investigated the prognostic value of urine osmolality from a nationwide prospective cohort of CKD patients in Korea. Low urine osmolality indicated a significantly greater risk of adverse renal outcomes, even after adjustment for various confounding factors. 

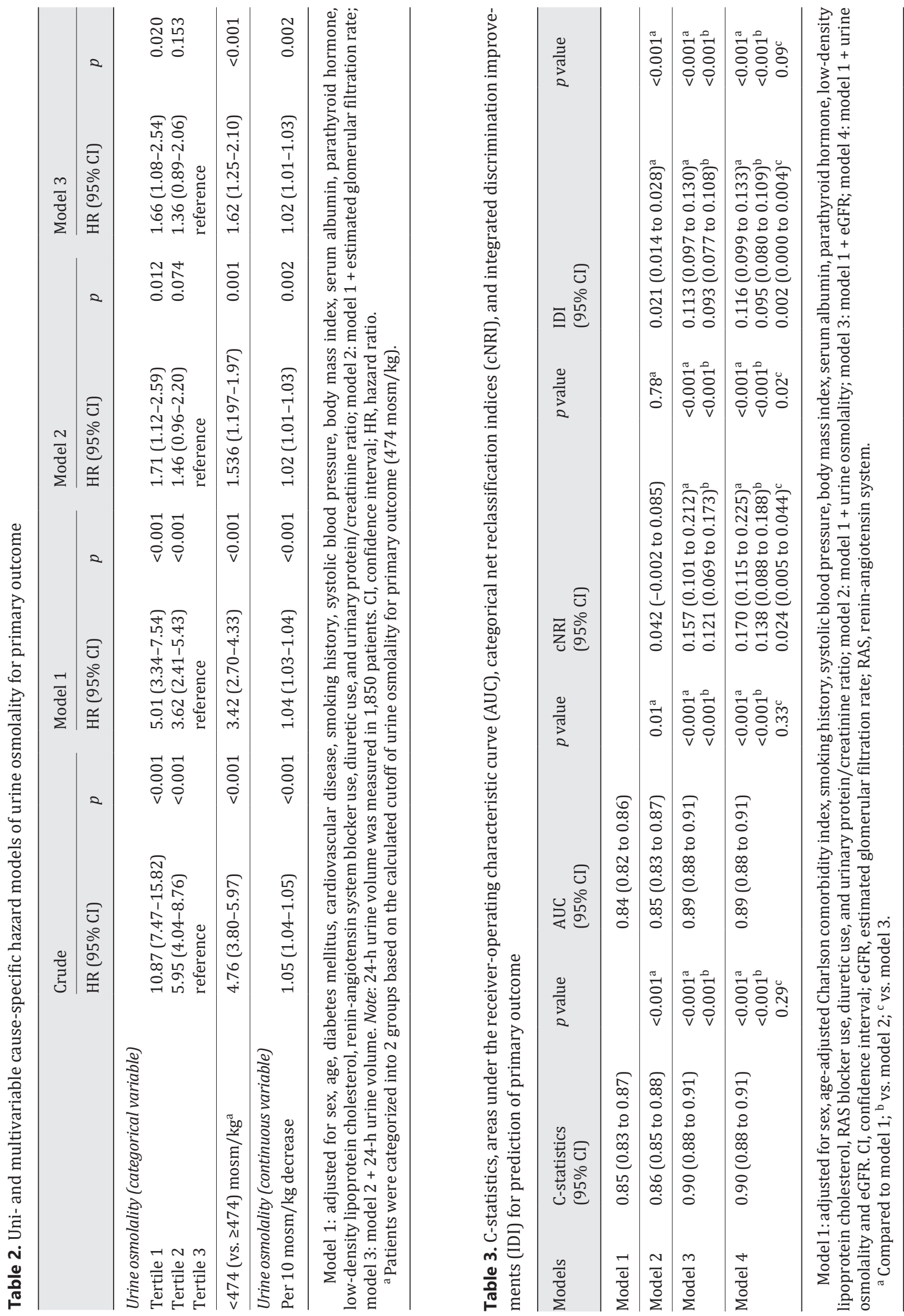


\section{Kidney \\ Blood Pressure \\ Research}

\begin{tabular}{l|l}
\hline Kidney Blood Press Res 2019;44:1089-1100 \\
\hline DOI: 10.1159/000502291 & $\begin{array}{l}\text { ○ 2019 The Author(s). Published by S. Karger AG, Basel } \\
\text { www.karger.com/kbr }\end{array}$ \\
\hline
\end{tabular}

Lee et al.: Urine Osmolality and Renal Outcome in CKD

Furthermore, subgroup analyses revealed that the detrimental effect of low urine osmolality on CKD progression was higher in patients with advanced-stage CKD. However, the predictive ability of urine osmolality for CKD progression did not surpass eGFR.

Although urine concentration is a unique function of the kidney, urine osmolality has received little attention in academic research and clinical practice. So far, only a few epidemiological studies explored the association of urine osmolality with kidney function decline in the CKD population [5-8]. Furthermore, given the conflicting results of previous studies [5-8], the prognostic value of urine osmolality on adverse renal outcomes has not been fully elucidated. In the post hoc analysis of the MDRD study [5], low urine osmolality was significantly associated with a faster GFR decline in patients with and without PKD. A recent study also showed that CKD stage 1-4 patients with lower urinary osmolality had a higher risk of end-stage renal disease but not mortality [8]. In line with these 2 studies, we showed that low urine osmolality was an independent risk factor for composite renal outcome, including a $50 \%$ decline in eGFR, necessity for dialysis, and renal transplantation. In contrast, a cohort study from CRISP reported that higher urine osmolality was correlated with a greater decline in GFR during the follow-up from years 1 to 6 in PKD patients [6]. Another study by Plischke et al. [7] demonstrated that a greater risk of dialysis initiation was found in patients with higher urine osmolality, and the risk ratio was roughly twofold in patients with CKD stages 1-4. Although the exact reason for these discrepancies is not clear, our subgroup analyses might provide possible explanations. In the PKD subgroup, the adverse effect of low urine osmolality remained significant in our study, which was similar to the MDRD post hoc study [5], but unlike that seen in the CRISP cohort study [6]. The mean baseline GFR of our PKD participants was $67.4 \pm 32.9 \mathrm{~mL} / \mathrm{min} / 1.73 \mathrm{~m}^{2}$. Although the mean GFR of the PKD subgroup was not known, the MDRD A study enrolled patients with a baseline GFR of $25-55 \mathrm{~mL} / \mathrm{min} / 1.73 \mathrm{~m}^{2}$ [5]. On the other hand, the CRISP cohort study included 241 autosomal dominant PKD patients aged 15-46 years with a creatinine clearance $>70 \mathrm{~mL} / \mathrm{min}$ at baseline [6]. Their mean eGFR was $89.1 \pm 27.7$ at baseline, and $72.1 \pm 28.3 \mathrm{~mL} / \mathrm{min} / 1.73 \mathrm{~m}^{2}$ after 6 years, suggesting faster progression of CKD in their participants [13]. Based on our subgroup analysis according to the CKD stages, the deleterious effect of low urine osmolality on kidney function was prominent in patients with eGFR $<60 \mathrm{~mL} / \mathrm{min} / 1.73 \mathrm{~m}^{2}$. Considering that one of the first clinical features of PKD is an impaired urine-concentrating capacity [14-17], we surmise that participants of the CRISP cohort were less likely to have tubular damage leading to a urine-concentrating defect. Plischke et al. [7] also included 273 patients with CKD stages 1-4, and they had a higher baseline urine osmolarity (median of 510, interquartile range, 414-622 mosm/L) than those in the MDRD post hoc study (270-334 mosm/L, depending on the protein diet group and PKD status) [5] or in our study. Although osmolality and osmolarity are not completely identical, we presume that the smaller number of subjects and a higher baseline urine osmolarity in the study by Plischke et al. [7] might limit evaluation of the prognostic value of low urine osmolality ( $<300 \mathrm{mosm} / \mathrm{L}$ ) properly. Furthermore, the level of adjustment such as 24 -h urine volume or use of diuretics significantly differed between the studies [5-7]. In the current study, a greater risk of low urine osmolality for the primary outcome remained significant after adjustment of 24-h urine volume and use of diuretics. However, a different level of adjustment and lack of subgroup analyses in the previous studies make it difficult to compare our results regarding the effect of urine volume or diuretic use. Considered together, we speculate that the heterogeneous characteristics of the studies, including a small number of subjects and the difference in the cause of primary kidney disease, kidney function, and statistical model construction might have contributed to the discrepancy between the previous studies and our study. Therefore, further prospective studies with a larger sample size across various CKD stages are necessary. 


\section{Kidney \\ Blood Pressure \\ Research}

\begin{tabular}{l|l}
\hline Kidney Blood Press Res 2019;44:1089-1100 \\
\hline DOI: 10.1159/000502291 & $\begin{array}{l}\text { ○ 2019 The Author(s). Published by S. Karger AG, Basel } \\
\text { www.karger.com/kbr }\end{array}$ \\
\hline
\end{tabular}

Lee et al.: Urine Osmolality and Renal Outcome in CKD

The mechanism underlying the adverse effect of low urine osmolality on CKD progression has not been fully understood. Low urine osmolality might be the result of a decline in renal function [5]. Tubular injury from progression of CKD can directly lead to decreased urine osmolality due to an impairment in the urine-concentrating function and/ or salt wasting. In our study, urine osmolality was relatively well maintained at 400-500 mosm $/ \mathrm{kg}$ in patients with CKD stage 3 and decreased below 400 mosm $/ \mathrm{kg}$ in those with CKD stage 4. Therefore, urine osmolality might not be sensitive to detect early kidney dysfunction. This is evidenced by the lower predictive ability of urine osmolality for CKD progression than that of eGFR. Alternatively, low urine osmolality might accelerate the decline in kidney function [5]. In fact, low urine osmolality might increase the intratubular urine volume and pressure, leading to a fibrogenic mechanism via stretch force $[18,19]$. In patients with PKD, increased intratubular pressure can promote cystic growth [13]. Meanwhile, the CRISP cohort investigators [6] and Plischke et al. [7] thought that high urine osmolality could reflect the vasopressin effect, which supports their finding of a detrimental effect of high urine osmolality on cyst growth in PKD. However, a study by Zittema et al. [20] revealed that PKD patients already had an impaired maximal urine-concentrating capacity in the early stages of disease, which is accompanied by increased plasma osmolality and vasopressin levels during water deprivation compared with healthy controls. Moreover, in another study involving advanced autosomal dominant PKD patients and immunoglobulin A nephropathy-matched controls, copeptin, a surrogate of vasopressin, was positively associated with plasma osmolality but inversely associated with urine osmolality at maximal urine concentration [17]. Based on the studies by Zittema et al. [17, 20], we surmised that reduced urine-concentrating capacity following tubular damage might lead to a decrease in urine osmolality and an increase in vasopressin, resulting in more kidney damage or cyst formation and expansion in PKD. This can partly explain why the association between low urine osmolality and adverse renal outcomes was evident in patients with eGFR $<60 \mathrm{~mL} /$ $\mathrm{min} / 1.73 \mathrm{~m}^{2}$ in our study. However, since we did not measure vasopressin or copeptin in our study, we could not confirm the exact underlying mechanism. Future studies are needed to understand the underlying mechanism by which low urine osmolality contributes to CKD progression.

This study has several limitations. Due to the observational nature of the study, the causal relationship between low urine osmolality and faster decline in kidney function cannot be affirmed by this study alone. Notably, this study demonstrated that there was a significant interaction between urinary osmolality and eGFR, in accordance with a recent study of Tabibzadeh et al. [8]. They suggest that the urine-concentrating ability is largely dependent on the kidney function. Furthermore, we clearly showed that prediction performance of urine osmolality was not greater than that of eGFR. Therefore, the prognostic value of urine osmolality should be interpreted in conjunction with kidney function. Second, this study did not contain any information regarding the therapeutic intervention to modulate urine osmolality. Third, as mentioned above, we could not explain the underlying mechanism between CKD progression and low urine osmolality. Notwithstanding these limitations, this study has also several strengths. We directly measured urine osmolality, mitigating the confounding effect of urinary electrolyte excretion or use of medication. Furthermore, our study included a large number of participants to investigate the association between urine osmolality and adverse renal outcomes in the CKD population from a well-examined nationwide prospective cohort. Although the residual confounding effects cannot be totally excluded, we constructed multivariable models using various confounders, such as diuretic use and 24-h urine volume. A sufficient number of participants with convincing data allowed us to analyze the effect of urine osmolality on adverse renal outcomes in detail. 


\section{Conclusion}

Low urine osmolality was an independent prognostic factor for disease progression in patients with CKD. This association was affected by kidney function but was particularly strong in patients with decreased eGFR. Our findings suggest that eGFR can modify the association between urine osmolality and CKD progression, and, thus, kidney function should be considered while interpreting the clinical significance of urine osmolality.

\section{Acknowledgments}

The KNOW-CKD study was conducted in 8 centers of Korea: the Seoul National University Hospital, Yonsei University Severance Hospital, Kangbuk Samsung Medical Center, Seoul St. Mary's Hospital, Gil Hospital, Eulji General Hospital, Chonnam National University Hospital, and Pusan Paik Hospital. The authors thank all of the KNOW-CKD investigators and Dr. Young $\mathrm{Su}$ Joo for support on statistics during the revision process.

\section{Statement of Ethics}

This study was conducted in accordance with the Declaration of Helsinki, and the study protocol was approved by the institutional review board at each participating hospital's clinical trial center. All participants provided written informed consent before participating in the study.

\section{Disclosure Statement}

The authors have no conflicts of interest to declare.

\section{Funding Sources}

This work was supported by the Research Program funded by the Korean Centers for Disease Control and Prevention (2011E3300300, 2012E3301100, 2013E3301600, 2013E3301601, 2013E3301602, and 2016E3300200).

\section{Author Contributions}

All authors confirmed they have contributed to the intellectual content of this paper and have met the following 4 requirements: (a) significant contributions to the study conception and design, and acquisition of data, or analysis and interpretation of data; (b) drafting or revising the article for intellectual content; (c) final approval of the published article; and (d) agreement to be accountable for all aspects of the article thus ensuring that questions related to the accuracy or integrity of any part of the article are appropriately investigated and resolved.

Conceptualization: M.J.L. and S.H.H.; statistical analysis: M.J.L., T.I.C, and S.H.H.; investigation: J.T.P., T.-H.Y., S.-W.K., and K.H.C.; supervision: J.L., Y.H.K., K.-H.O., S.W.L, S.W.K., and C.A.; writing-original draft: M.J.L. and S.H.H. 


\section{References}

1 Keith DS, Nichols GA, Gullion CM, Brown JB, Smith DH. Longitudinal follow-up and outcomes among a population with chronic kidney disease in a large managed care organization. Arch Intern Med. 2004 Mar;164(6):659-63.

2 Collins AJ, Foley RN, Herzog C, Chavers B, Gilbertson D, Ishani A, et al. United States Renal Data System 2008 Annual Data Report. Am J Kidney Dis. 2009 Jan;53(1 Suppl):S1-374.

3 Jin DC, Yun SR, Lee SW, Han SW, Kim W, Park J, et al. Current characteristics of dialysis therapy in Korea: 2016 registry data focusing on diabetic patients. Kidney Res Clin Pract. 2018 Mar;37(1):20-9.

4 Foley RN, Parfrey PS, Sarnak MJ. Clinical epidemiology of cardiovascular disease in chronic renal disease. Am J Kidney Dis. 1998 Nov;32(5 Suppl 3):S112-9.

5 Hebert LA, Greene T, Levey A, Falkenhain ME, Klahr S. High urine volume and low urine osmolality are risk factors for faster progression of renal disease. Am J Kidney Dis. 2003 May;41(5):962-71.

6 Torres VE, Grantham JJ, Chapman AB, Mrug M, Bae KT, King BF Jr, et al.; Consortium for Radiologic Imaging Studies of Polycystic Kidney Disease (CRISP). Potentially modifiable factors affecting the progression of autosomal dominant polycystic kidney disease. Clin J Am Soc Nephrol. 2011 Mar;6(3):640-7.

7 Plischke M, Kohl M, Bankir L, Shayganfar S, Handisurya A, Heinze G, et al. Urine osmolarity and risk of dialysis initiation in a chronic kidney disease cohort—a possible titration target? PLoS One. 2014 Mar;9(3):e93226.

8 Tabibzadeh N, Wagner S, Metzger M, Flamant M, Houillier P, Boffa JJ, et al.; NephroTest Study Group. Fasting urinary osmolality, CKD progression, and mortality: A prospective observational study. Am J Kidney Dis. 2019 May;73(5):596-604.

9 Oh KH, Park SK, Park HC, Chin HJ, Chae DW, Choi KH, et al.; Representing KNOW-CKD Study Group. KNOW-CKD (KoreaN cohort study for Outcome in patients With Chronic Kidney Disease): design and methods. BMC Nephrol. 2014 May; 15(1):80.

10 Charlson M, Szatrowski TP, Peterson J, Gold J. Validation of a combined comorbidity index. J Clin Epidemiol. 1994 Nov;47(11):1245-51.

11 Levey AS, Stevens LA, Schmid CH, Zhang YL, Castro AF 3rd, Feldman HI, et al.; CKD-EPI (Chronic Kidney Disease Epidemiology Collaboration). A new equation to estimate glomerular filtration rate. Ann Intern Med. 2009 May; 150(9):604-12.

12 Chapter 1: definition and classification of CKD. Kidney Int Suppl (2011). 2013 Jan;3(1):19-62.

13 Grantham JJ. The etiology, pathogenesis, and treatment of autosomal dominant polycystic kidney disease: recent advances. Am J Kidney Dis. 1996 Dec;28(6):788-803.

14 Gabow PA, Kaehny WD, Johnson AM, Duley IT, Manco-Johnson M, Lezotte DC, et al. The clinical utility of renal concentrating capacity in polycystic kidney disease. Kidney Int. 1989 Feb;35(2):675-80.

15 Fick GM, Gabow PA. Hereditary and acquired cystic disease of the kidney. Kidney Int. 1994 Oct;46(4):951-64.

16 Torres VE, Harris PC, Pirson Y. Autosomal dominant polycystic kidney disease. Lancet. 2007 Apr;369(9569): 1287-301.

17 Zittema D, Casteleijn NF, Bakker SJ, Boesten LS, Duit AA, Franssen CF, et al. Urine Concentrating Capacity, Vasopressin and Copeptin in ADPKD and IgA Nephropathy Patients with Renal Impairment. PLoS One. 2017 Jan; 12(1):e0169263.

18 Gruden G, Thomas S, Burt D, Zhou W, Chusney G, Gnudi L, et al. Interaction of angiotensin II and mechanical stretch on vascular endothelial growth factor production by human mesangial cells. J Am Soc Nephrol. 1999 Apr;10(4): 730-7.

19 Riser BL, Cortes P, Heilig C, Grondin J, Ladson-Wofford S, Patterson D, et al. Cyclic stretching force selectively up-regulates transforming growth factor-beta isoforms in cultured rat mesangial cells. Am J Pathol. 1996 Jun; 148(6):1915-23.

20 Zittema D, Boertien WE, van Beek AP, Dullaart RP, Franssen CF, de Jong PE, et al. Vasopressin, copeptin, and renal concentrating capacity in patients with autosomal dominant polycystic kidney disease without renal impairment. Clin J Am Soc Nephrol. 2012 Jun;7(6):906-13. 